\title{
Histological findings on jaw osteonecrosis associated with bisphosphonates (BONJ) or with radiotherapy (ORN) in humans
}

\author{
DANIELA CARMAGNOLA ${ }^{1}$, ELENA CANCIANI ${ }^{1}$, DAVIDE SOZZI $^{2}$, FEDERICO BIGLIOLI $^{3}$, \\ LAURA MONEGHINI $^{4}$ \& CLAUDIA DELLAVIA ${ }^{1}$
}

${ }^{1}$ Department of Biomedical, Surgical and Dental Sciences, Università degli Studi di Milano, Italy, ${ }^{2}$ Department of
Neurosciences and Biomedical Technologies, Università degli Studi di Milano-Bicocca, Unit of Maxillo-Facial Surgery,
Ospedale San Gerardo di Monza, Italy, ${ }^{3}$ Department of Health Sciences, Unit of Maxillo-Facial Surgery, and
${ }^{4}$ Department of Health Sciences, Division of Pathology, A.O. San Paolo, Università degli Studi di Milano, Italy

\begin{abstract}
Objective. To describe the histological features of bone tissue harvested from patients affected by jaw osteonecrosis associated with bisphoshponates (BONJ) or with radiotherapy (ORN), in undecalcified ground sections. Materials and methods. Sixteen bone tissue samples from 14 patients with BONJ and two patients with ORN were processed in order to obtain both ground, undecalcified sections and decalcified sections. The sections underwent histometric and morphometric analysis. Results. Bone tissue samples obtained from patients with BONJ or ORN of the jaws shared some histological characteristics. Common histological features included the loss of bone architecture, the absence of a proper Haversian system and proper marrow spaces, the presence of necrotic spots of non-mineralized tissue, areas of empty osteocytic lacunae next to areas of hypercellularity, the presence of resorption pits with rare osteoclast-like cells and the presence of bacteria and of an inflammatory infiltrate. A violet rib of tissue characterized by large resorption pits facing was frequently observed between the mineralized bone and the inflammatory infiltrate. Conclusions. The histological features of BONJ and ORN are similar and resemble those of osteomyelitis. Even though it is not clear whether infection is the cause or consequence of bone exposure, inflammatory cells, bacteria or their products may have a massive, direct lytic effect on bone tissue challenged by bisphosphonates.
\end{abstract}

Key Words: bisphosphonates, osteonecrosis of the jaws, ORN

\section{Introduction}

Bisphosphonates are medications widely used in the management of osteoporosis, Paget's disease and to prevent skeletal complications of multiple myeloma and bone metastases from solid cancer [1]. They are taken up in the skeleton at sites with increased bone remodeling [2]. Bisphosphonates treatment rapidly decreases bone resorption and progressively decreases bone formation by inhibiting osteoclastic activity and consequently affecting osteoclastic-induced osteoblastic activity. Bone turnover is therefore suppressed $[3,4]$. Angiogenesis is reduced due to the depression of the blood flow and a marked decrease in the vascular endothelial growth factor [5]. Epithelial keratinocytes are also inhibited due to the interference of bisphosphonates with p63-positive progenitor cells in the basal layer of the oral epithelium, resulting in a delayed oral epithelial healing [3]. Overall, the bone healing capacity is reduced [5]. A complication observed following treatment with bisphosphonates is osteonecrosis of the jaws (ONJ). According to the American Association of Oral and Maxillo-Facial Surgeons (AAOMS) [6], jaw osteonecrosis associated with bisphoshponates (BONJ) is defined as 'exposed bone in the maxillofacial region that has persisted for more than 8 weeks, with current or previous treatment with a bisphosphonate and no history of radiation therapy to the jaws' [6]. Different stages of BONJ are described, ranging from stage 0 (no bone exposure) to stage 3 (bone exposure with pain and infection, extra-oral fistula, exposed necrotic bone

Correspondence: Daniela Carmagnola, Department of Human Morphology and Biomedical Sciences 'Città Studi', Università degli Studi di Milano, Via Mangiagalli 31, 20133 Milan, Italy. Tel: +390250315405. Fax: +390250315387. E-mail: daniela.carmagnola@unimi.it 
extending beyond the region of the alveolar bone, pathological fracture, osteolysis extending to the inferior border or oro-antral communication). BONJ is usually treated in a conservative way unless mobile sequestrum (stage 3) is present and prompts for removal. The reasons behind bone exposure are not completely clear, but dento-alveolar surgery and extractions have been suggested as risk factors for developing BONJ, even months after surgery, suggesting that a lack of repair due to the suppression of bone turnover plays an important role in the pathogenesis of BONJ. From a microbiological point of view, Actinomycetes species and Eikenella corrodens were isolated from such lesions [7-10]. Microscopically, BONJ necrotic bone was described as composed of non-viable bone trabeculae that have empty lacunae often with ragged, scalloped borders. Chronic or acute inflammatory cells, abscesses or bacteria may be present [4]. A variant of BONJ that does not manifest with necrotic bone exposure or mucosal fenestration was recently described and should be carefully considered in patients with persistent jaw bone pain, bone enlargement, gingival swelling or sinus tract in the absence of relevant dental disease. Up to half of the patients with nonexposed osteonecrosis may never progress to develop frank bone exposure and up to one third would show no obvious radiological abnormalities [11].

Jaw osteonecrosis associated with radiotherapy $(\mathrm{ORN})$ is characterized by persisting bone exposure and may become evident long after therapy, although recovery of bone-regenerative properties has been proven 1-year after radiation. Radiated tissue is hypovascular, hypocellular and hypoxic $[4,12]$. ORN occurs more frequently in the mandible and the risk of developing it is increased by increasing radiation dose, tumor's close proximity to bone, poor oral health and the type of treatment the patient undergoes. ORN shares similar features with BONJ. Necrotic bone is exposed with little or no symptoms at the beginning. Pain, abscess and fistula formation might occur with time. Therapy includes the use of antibiotics and hyperbaric oxygen therapy (HBO) [4]. According to the outcome of HBO therapy, ORN can be classified as stage 1 (exposed bone), stage 2 (no response to HBO therapy) or stage 3 (pathological fracture), oro-cutaneous fistula or osteolysis to pathological fracture, oro-cutaneous fistula or osteolysis to the inferior border of the mandible) [4]. Microbiological studies have shown the presence of Actinomycetes species in biopsy specimens from ORN $[13,14]$ that were associated with an increased risk of treatment failure or the need for prolonged treatment. Other studies performed in deep bony lesions showed anaerobic bacteria with a predominance of Porphyromonas gingivalis, as well as Actinomycetes species, Prevotella species and Fusobacterium nucleatum [15].
Despite the many papers published on different features of BONJ in the last decade, the puzzle of its pathogenesis is still incomplete. Some reports describe the histological characteristics of bone tissue affected by BONJ and ORN, mainly on decalcified sections. The aim of this study was to characterize the histological features of bone tissue obtained by patients affected by BONJ and ORN on bone samples processed for ground sectioning, as ground sections preserve the mineral component and may, therefore, be suitable to study conditions affecting the bone.

\section{Materials and methods}

For this purpose, 14 bone tissue samples were obtained from 14 patients in treatment with bisphosphonates with BONJ and two samples from two patients with ORN referred to a specialist for case management. The centers involved in the present study were the Unit of Maxillo-Facial Surgery, Department of Medicine, Surgery and Dentistry, A.O. San Paolo, Università degli Studi di Milano and the Unit of Maxillo-Facial Surgery, Department of Neurosciences and Biomedical Technologies, Università degli Studi di Milano-Bicocca, Ospedale San Gerardo di Monza.

Biologic material and data were treated according to the Helsinki declaration. No additional procedure to routine standard care was carried out in the patients. The study received ethical approval by the Department of Human Morphology and Biomedical Sciences 'Città Studi', Università degli Studi di Milano, Milan, Italy. All participants signed an informed consent form concerning the treatment protocol.

The characteristics of the patients are described in Table I. All patients on bisphosphonates showed stage 3 BONJ and needed, therefore, surgical debridement of the lesion. One week before surgery, the patients received professional oral hygiene (scaling and root planning) and were instructed to mouthrinse with $0.2 \%$ chlorhexidine twice a day for 2 weeks. Further, they were given amoxicillin $1 \mathrm{~g}$ 3-times daily for 14 days, starting 4 days before surgery [6]. The surgical procedure was performed under local or general anesthesia. Briefly, when necessary a flap was raised to expose the affected area, the sequestrum or mobile necrotic bone were removed, bone curettage was carried out, the area was flushed with saline solution and the flap was sutured.

The two patients with ORN had bone exposure that did not heal with antibiotics and HBO therapy and were, therefore, scheduled for surgery. The surgical protocol was similar to the one for BONJ patients, except for the fact that bone was removed until 'normal' bleeding was observed from the margins.

The tissue samples were immediately placed in formalin and processed for ground sectioning at the 
Table I. Characteristics of the sample population.

\begin{tabular}{lllllll}
\hline & Age & $\begin{array}{c}\text { Bisphosphonates } \\
\text { duration (months) }\end{array}$ & \multicolumn{1}{c}{ Treatment } & Area of necrosis & Pathology \\
\hline 1 & F & 59 & 72 & Alendronate & Mandible & Osteoporosis \\
2 & F & 85 & 240 & Alendronate & Maxilla & Osteoporosis \\
3 & F & 77 & 120 & Alendronate & Mandible & Osteoporosis \\
4 & F & 50 & 110 & Alendronate & Maxilla & Osteoporosis \\
5 & F & 57 & 41 & Zoledronic acid & Mandible & Breast cancer \\
6 & M & 65 & 24 & Zoledronic acid & Mandible & Multiple myeloma \\
7 & M & 59 & 17 & Zoledronic acid & Mandible & non-Hodgkin Lymphoma \\
8 & M & 76 & 19 & Zoledronic acid & Mandible & Prostatic cancer \\
9 & M & 58 & 41 & Zoledronic acid & Mandible & Multiple myeloma \\
10 & M & 76 & 12 & Zoledronic acid & Mandible & Kidney cancer \\
11 & M & 76 & 22 & Zoledronic acid & Mandible & Prostatic cancer \\
12 & F & 73 & $180+10$ & Alendronate + Zoledronic acid & Maxilla & Multiple myeloma \\
13 & M & 61 & 9 & Zoledronic acid & Mandible & Kidney cancer \\
14 & F & 66 & 38 & Zoledronic acid & Mandible & Breast cancer \\
15 & M & 57 & - & Radiation therapy & Mandible & Oral cancer \\
16 & M & 65 & - & Radiation therapy & Mandible & Oral cancer \\
\hline & & & & &
\end{tabular}

Department of Human Morphology and Biomedical Sciences 'Città Studi', Università degli Studi di Milano and for paraffin sectioning at the Division of Pathology of the Department of Medicine, Surgery and Dentistry, Università degli Studi di Milano.

\section{Histological processing}

The central portion of each sequestrum was processed for ground sectioning according to the method of Donath and Breuner [16], in order to observe the structure of the bone samples without removing the mineral component. Briefly, the specimens were fixed in $10 \%$ formalin, dehydrated by increasing ethanol concentrations and embedded in Kulzer Technovit 7200 VLC $^{\circledR}$ (Bio-Optica, Milano, Italy) resin. The cores were sliced longitudinally and subsequently reduced by micro-grinding and polishing to an even thickness of $80 \mu \mathrm{m}$ (Micromet \& LS2 ${ }^{\circledR}$, Remet, Bologna, Italy). The sections were stained with Toluidine blue/Pyronin Y (Sigma-Aldrich, St. Louis, $\mathrm{MO})$. Such staining colors bone in varying shades of blue according to the degree of bone mineralization. Two central sections per specimen were used for analysis.

Furthermore, $5 \mu \mathrm{m}$ thick paraffin sections were obtained by decalcifying the bone biopsies for $3 \mathrm{~h}$ in Histo-Decal (Histo Line Laboratories, Milano, Italy). The sections were stained with hematoxylin and eosin.

The sections were observed using a Nikon light microscope (Eclipse E600 ${ }^{\circledR}$, Nikon, Tokyo, Japan) equipped with a calibrated digital camera (DXM1200 ${ }^{\circledR}$, Nikon). All aspects of each section were photomicrographed at a magnification of $20 \times$ and merged by means of image analysis software (Rhinoceros NURBS modeling for Windows, version 3.0, McNeel, Seattle, WA) to obtain the whole image of the bone sample. Histometric linear measurements were performed on the images in order to estimate the percentage of the bone margin characterized by resorption pits in the necrotic areas of the samples. Morphometric measurements were performed in order to quantify the proportion of the sample characterized by medullary spaces, cortical component and blood vessels, using a point-counting procedure with a 100 test point grid, at a magnification of $40 \times$.

For each parameter, mean values and standard deviations were calculated separately for three groups: oral bisphosphonates, intravenous bisphosphonates and ORN.

\section{Results}

Sixteen specimens from 16 patients were processed. Four patients were on oral bisphosphonates treatment for osteoporosis, 10 patients were on Zometa for malignant tumors, while two patients had oral cancer and had been treated with radiotherapy of the head (Table I). At gross histological observation, the specimens from oral and intravenous bisphosphonates as well as those from ORN did not show relevant differences. Thirteen specimens were of mandibular origin and three were of maxillary origin.

Overall, the microscopic architecture of bone and soft tissue was damaged or even lost, although in the ORN samples it seemed to be better preserved than in the BONJ samples (Figure 1). Areas of mineralized tissue were observed near areas of non-mineralized tissue that could represent either residual marrow 

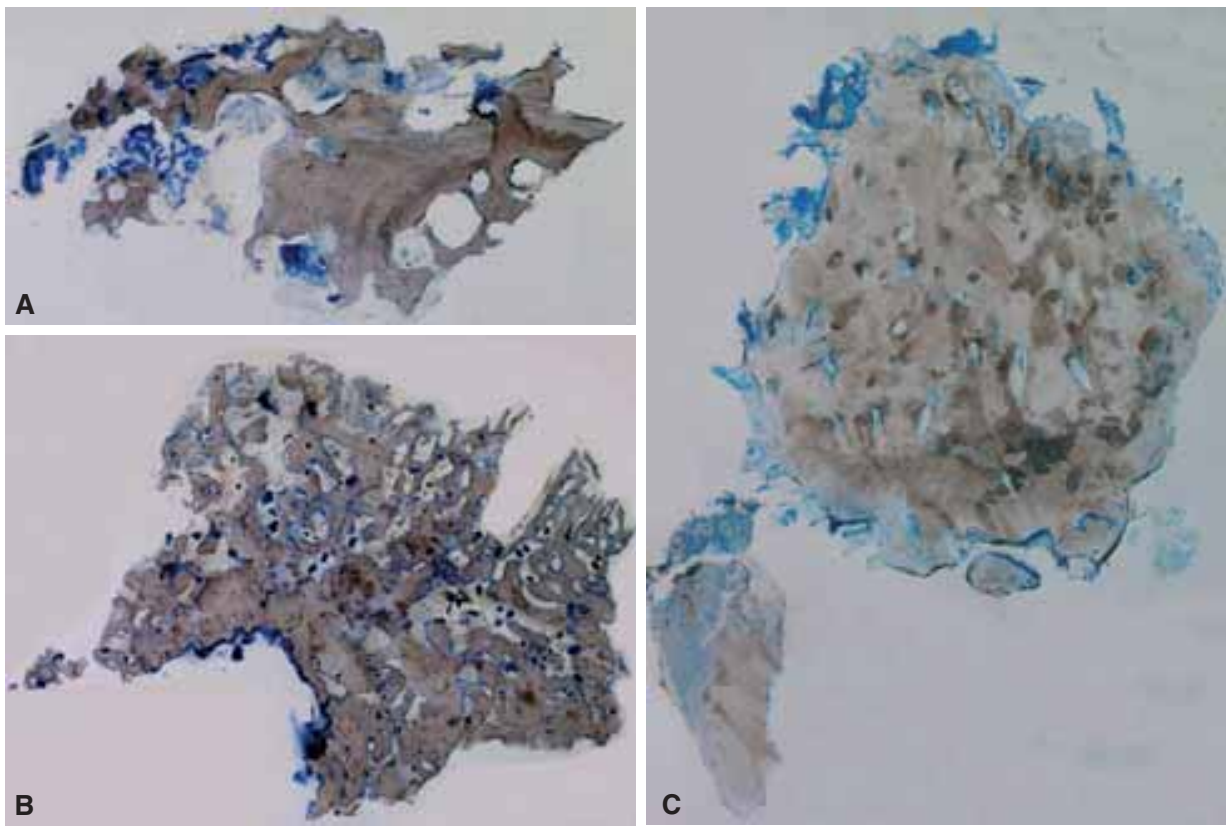

Figure 1. Panel of images representing three typologies of osteonecrosis. (A) Oral bisphosphonate; (B) intravenous bisphosphonates (Zometa); (C) radiosteonecrosis. Undecalcified sections; original magnification $5 \times$, Toluidine Blue and Pyronine Y staining.

spaces, necrotic bone or necrotic connective tissue (Figure 2).

Concerning the mineralized component, the typical Haversian system with a central canal was seldom observed and marrow spaces were virtually absent. The bone was rather composed of fragmented osteons surrounded by sparse reversal lines. Areas characterized by empty lacunae were observed next to areas with large hypercellularity. Very few vessels, sometimes with thick walls (Figure 3), were detected both in the mineralized and non-mineralized tissue. Most of the BONJ samples' bone profile was irregular, indented and characterized by resorption pits rich in neutrophil granulocytes, while osteoblasts

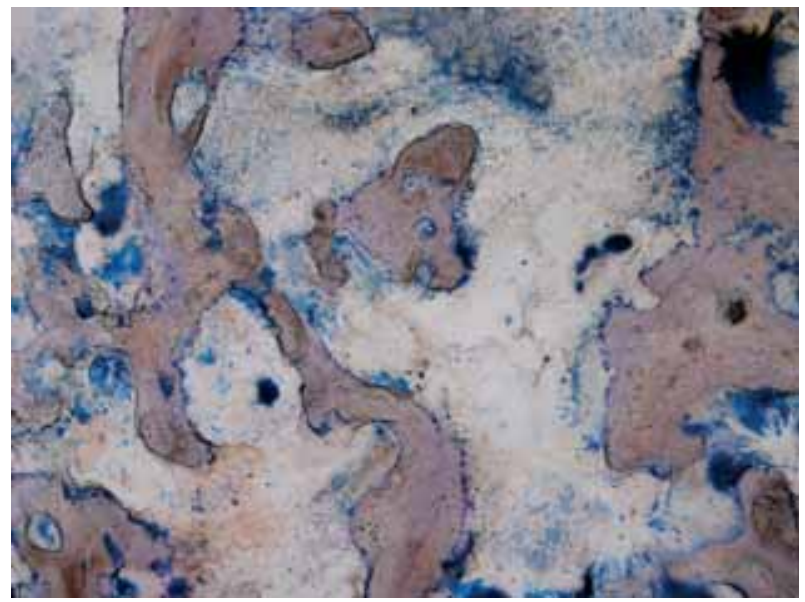

Figure 2. Photomicrography showing the presence of areas of nonmineralized tissue facing mineralized tissue. Bone and soft tissue appear damaged and unorganized with an irregular profile. Undecalcified section; total magnification $100 \times$, Toluidine Blue and Pyronine Y staining. and osteoclasts-like cells were seldom identified (Figure 4). This feature was also observed in the osteoradionecrotic bone, although to a lesser extent. An inflammatory infiltrate was often observed at the border between mineralized and non-mineralized tissue, mainly characterized by granulocytes, plasma cells and occasionally lymphocytes (Figure 5). A violet rib of tissue, characterized by large resorption pits with few osteoclast-like cells, was frequently observed between the mineralized bone and the inflammatory infiltrate front (Figure 5B). The aspect of all specimens suggested bacterial infiltration (Figure 6).

The non-mineralized component didn't show the typical characteristics of connective and marrow

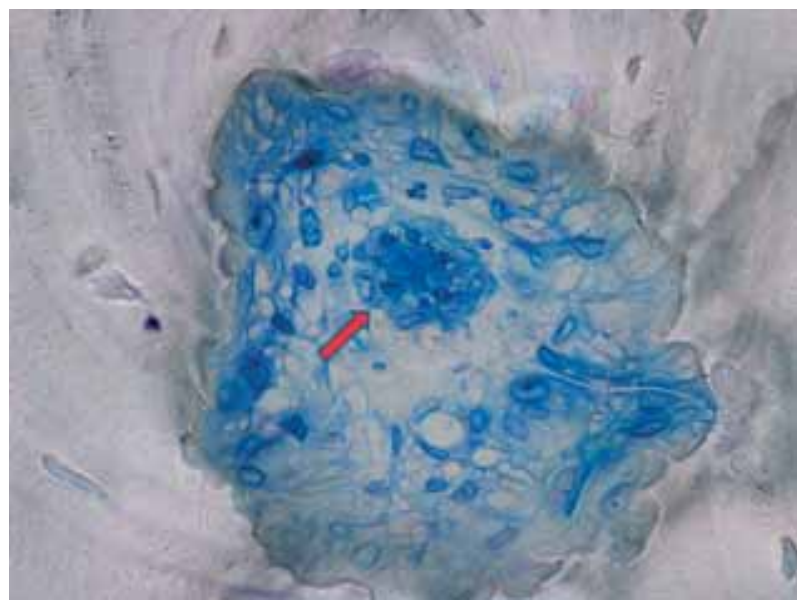

Figure 3. Photomicrography representing a blood vessel with thickened endothelial walls and activated endothelial cells (arrows). Undecalcified section; total magnification $600 \times$, Toluidine Blue and Pyronine Y staining. 

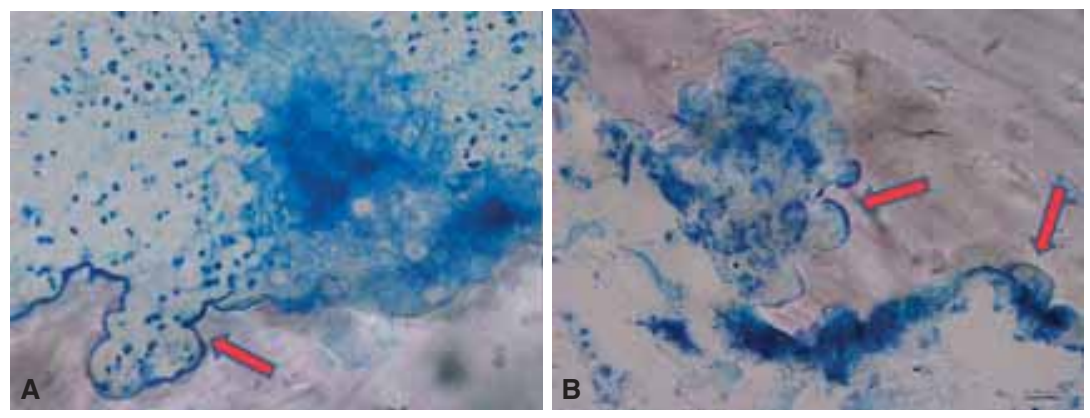

Figure 4. Panel showing the pattern of the bone profile with resorption pits (arrows). Undecalcified section; total magnification $600 \times$, Toluidine Blue and Pyronine Y staining.
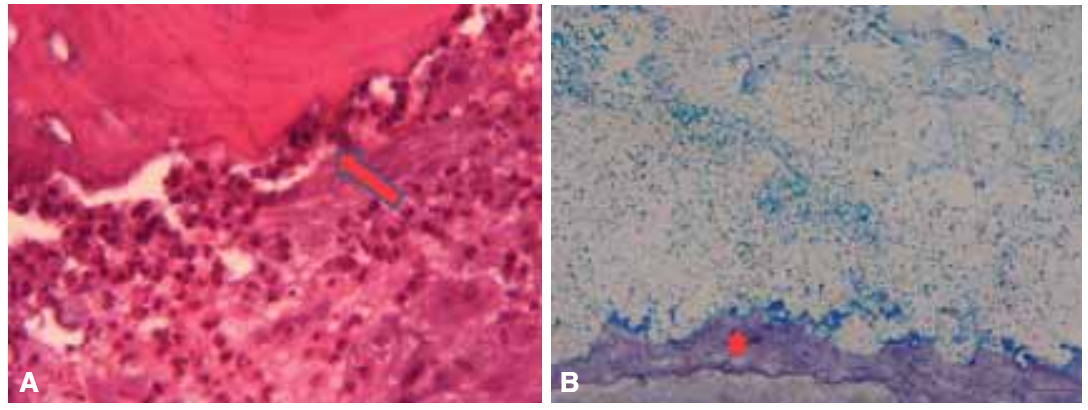

Figure 5. Photomicrographies showing an inflammatory infiltrate. In (A) granulocytes and plasmacells can be recognized in proximity of the bone and connective tissue (arrows). In (B) the violet tissue (asterisk) between soft tissue and bone might be the result of a lytic process caused by bacteria and cells. (A) Decalcified section; total magnification $1000 \times$, Hematoxylin and Eosin staining. (B) Undecalcified section; total magnification $200 \times$, Toluidine Blue and Pyronine Y staining.

spaces, as adipocytes were absent, few residual collagen fibers were distributed irregularly and acellular necrotic debris occupied most of the space.

Under polarized light (Figure 7), the mineralized tissue showed different histological aspects advancing from 'normal' to necrotic areas. In the necrotic portions the bone architecture was lost while, where the tissue was 'normal', a regular organization of the fibers could be appreciated. Atrophic or hyperplasic

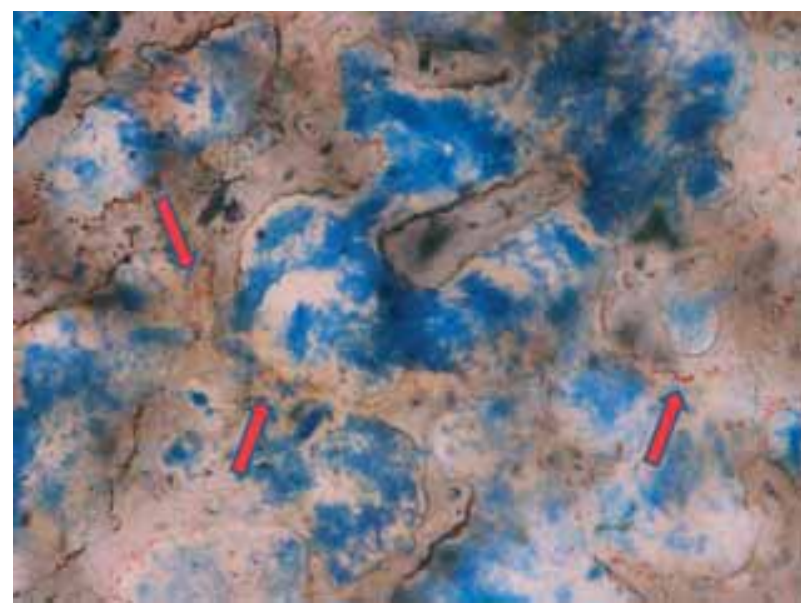

Figure 6. Photomicrography showing irregular trabecular bone and soft tissue with bacteria stained in brown-orange (arrows). Undecalcified section; total magnification $100 \times$, Toluidine Blue and Pironine Y staining. epithelial-like cells were sometimes observed, probably deriving from the disrupted epithelial tissue surrounding the lesion.

Resorption pits occupied $83.7 \% \pm 18.2 \%$ of the bone margin in the necrotic portions of the intravenous bisphosphonates samples. The corresponding values for oral bisphosphonates were and $89.7 \% \pm$ $16.5 \%$ and $42.5 \% \pm 3.5 \%$ for the two ORN specimens (Table II).

In the intravenous bisphosphonates group the medullary spaces occupied $31 \% \pm 10 \%$, while the cortical portion was $69 \% \pm 10 \%$. The corresponding values for oral bisphosphonates were $46 \% \pm 4 \%$ and $54 \% \pm 4 \%$ and for ORN $29 \% \pm 2 \%$ and $71 \% \pm 2 \%$ (Table II).

Blood vessels proportion was $2.1 \% \pm 3.7 \%$ for oral bisphosphonates, $3.0 \% \pm 5.7 \%$ for intravenous bisphosphonates and $2.5 \% \pm 5.0 \%$ for ORN (Table II).

\section{Discussion}

Bone tissue samples obtained from patients with BONJ or ORN shared similar histological characteristics, like the absence of a proper Haversian system and proper marrow spaces, the presence of necrotic spots of non-mineralized tissue, areas of empty osteocytic lacunae next to areas of hypercellularity, the presence of resorption pits (to a larger extent in BONJ samples) with rare osteoclast-like cells, the 


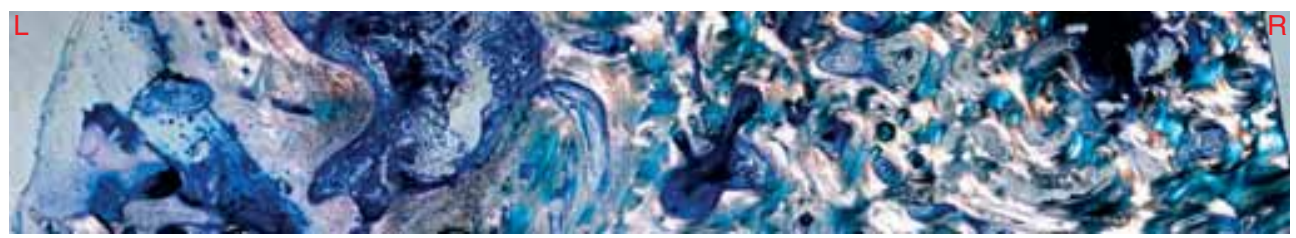

Figure 7. Photomicrography. From right $(\mathrm{R})$ to left $(\mathrm{L})$, the image shows a progressive loss of birefraction, suggesting a reduction of bone vitality. Polarized light. Undecalcified section; original magnification $5 \times$.

presence of bacteria and of an inflammatory infiltrate. It has to be underlined that the comparison included only two osteradionecrosis cases vs 14 BONJ samples. The reason is that in our centers ORN has become a rare event, probably due to an improvement in the administration of radiotherapy in the last years and, therefore, only two cases occurred in the study time span. Nevertheless, the histological similarities in the two groups prompted us not to omit the ORN specimens. In line with our findings, Almazrooa and Woo [4] reported that necrotic bone, regardless of its etiology, is microscopically composed of non-viable trabeculae with empty lacunae and ragged, scalloped borders associated with the presence of chronic or acute inflammatory cells, abscesses or bacteria. Bisphosphonates bone lesions are mainly confined to the jawbones, anatomical sites frequently subjected to trauma and manipulation for dental procedures [17]. A disruption of the integrity of the oral mucosal epithelium together with a reduced local bone response to traumas could be crucial for the of development infections like osteomyelitis [18] that share common features with BONJ. Although it is not clear whether infection is the cause or consequence of bone exposure, bacterial contamination was detected in all samples of the present study in proximity of the mineralized tissue's necrotic portion. In this respect, it has to be underlined that all tissue samples included in the present study came from patients with advanced exposed osteonecrosis and, therefore, infection may well have represented a consequence of long-term exposure of necrotic bone. On the other hand, reports exist that have identified bacterial species such as Fusobacterium, Eikenella, Bacillus, Actinomyces, Staphylococcus and Streptococcus in ORN $[15,19]$ and $\mathrm{BONJ}$ lesions, suggesting a direct role of biofilms in BONJ onset and bone resorption [7,20-23]. Furthermore, it has been shown that several species of bacteria produce mediators like lipopolysaccharides that could cause alveolar bone destruction [24]. Sedghizadeh et al. [25] observed a large number of microbial pathogens in the resorption pits on the bone surface of bone samples obtained from BONJ lesions. The authors mentioned that the depth of the pits varied with the amount of bacteria present and that osteoclasts were not identified in or near the resorption pits, suggesting a direct role for biofilms in the resorption process. Hansen et al. [19] also found Actinomyces colonies both in BONJ and ORN samples, most commonly detected at the site of necrotic bone exhibiting remarkable signs of erosion. An interesting finding in the present study was the frequent observation on the ground sections of a rib of tissue between the mineralized bone and the inflammatory infiltrate front in BONJ samples. Considering the above-reported findings, the violet rib of tissue described in the present study may be the expression of the lytic process of bacteria on the bone profile. The common features among BONJ, ORN and osteomyelitis of the jaws may be summarized as follows: when oral bacteria spread into a 'vulnerable' bone, i.e. a tissue influenced by bisphosphonates or radiotherapy, or in a compromised patient, the impact of microorganisms and their mediators may result in resorption pits and bone necrosis. In this picture, the violet rib might represent the transition between bacterial invasion and necrosis. Bacteria seem, therefore, to play a crucial role, regardless of the cause of the bone tissue weak response to infection.

In contrast to our findings, some authors have observed numerous osteoclasts both in BONJ and radionecrotic lesions, localized in close contact to bone lacunae [19]. In a histological study on bone samples from healthy patients with osteoporosis on alendronate for up to 3 years, Weinstein et al. [26] observed an increase in the number of osteoclasts,

Table II. Perimeter occupied by Howship-like lacunae (\%) and tissue composition (\% of medullary spaces and cortical bone) in the different groups.

\begin{tabular}{lccc}
\hline & Oral bisphosphonates & Intravenous bisphosphonates & Osteoradionecrosis \\
\hline Perimeter occupied by resorption pits (\%) & $89.7 \pm 16.5$ & $83.7 \pm 18.2$ & $42.5 \pm 3.5$ \\
Medullary spaces (\%) & $46 \pm 4$ & $31 \pm 10$ & $29 \pm 2$ \\
Cortical portion (\%) & $54 \pm 4$ & $69 \pm 10$ & $71 \pm 2$ \\
Blood vessels (\%) & $2.1 \pm 3.7$ & $3.0 \pm 5.7$ & $2.5 \pm 5.0$ \\
\hline
\end{tabular}


organized in giant cells undergoing protracted apoptosis, compared to control patients under placebo. Major differences between the present study and the one by Weinstein et al. include the type of bisphosphonate, the fact that our patients were affected by BONJ and that bone samples in the study by Weinstein et al. were harvested from the iliac crest.

In the present study few irregular vessels, often with thick walls, were observed both in the mineralized and non-mineralized tissue of all samples. Radiated tissue is hypovascular as vascular obliteration is the consequence of radiation-induced endarteritis followed by hyalinized narrowing of the vessels $[27,28]$. Concerning bisphosphonates, according to some authors such medications induce obliteration of the regional blood vessels leading to avascular bone necrosis [29], have anti-angiogenic effects and cause ischemia of the jawbone $[21,30]$ by inhibiting endothelial function in vitro and in vivo [31] and decreasing the levels of vascular endothelial growth factor in oncologic patients [32]. On the contrary, other authors have observed that the necrotic areas of BONJ lesions harbor a normal vasculature $[19,33]$. In a study aimed at comparing the histological characteristics of BONJ and osteoradionecrotic lesions, Hansen et al. [19] did not observe a significant reduction of the capillaries in BONJ lesions and described an increased cellularity both in the intima and media of segmental arteries. In ORN lesions, the intima and media were hyalinized and exhibited fewer cells. An interesting contribution to the understanding of BONJ's pathogenesis was recently provided by Favia et al. [17]. In a histological study on decalcified and non-decalcified sections of human bone samples obtained from patients with BONJ, the authors suggested that in bone treated with bisphosphonates, appositional osteogenesis occurs without concurrent remodeling or resorption, resulting in a lack of the vascular compartment expansion and depletion of marrow spaces. Such events would lead to ischemic necrosis of the bone tissue that, together with bacterial infection, would eventually result in BONJ.

In conclusion, the pathogenesis of BONJ seems to include multiple mechanisms that are still not completely understood and histological studies may contribute to complete the puzzle. We observed that BONJ and ORN share similar features and resemble common osteomyelitis. Even though it is not clear whether infection is cause or consequence of bone exposure, the rib of tissue that was observed in the resorption areas of the necrotic bone samples in the present study may be the expression of a massive, direct lytic process of bacteria on the profile of bone tissue challenged by bisphosphonates.

Declaration of interest: The authors report no conflicts of interest. The authors alone are responsible for the content and writing of the paper.

\section{References}

[1] Landesberg R, Cozin M, Cremers S, Woo V, Kousteni S, Sinha $S$, et al. Inhibition of oral mucosal cell wound healing by bisphosphonates. J Oral Maxillofac Surg 2008; 66:839-47.

[2] Cremers S, Papapoulos S. Pharmacology of bisphosphonates. Bone 2011;49:42-9.

[3] Scheller EL, Baldwin CM, Kuo S, D'Silva NJ, Feinberg SE, Krebsbach PH, et al. Bisphosphonates inhibit expression of p63 by oral keratinocytes. J Dent Res 2011;90:894-9.

[4] Almazrooa SA, Woo SB. Bisphosphonate and nonbisphosphonate associated osteonecrosis of the jaw. A review. J Am Dent Assoc 2009;140:864-75.

[5] Cheng A, Daly CG, Logan RM, Stein B, Goss AN. Alveolar bone and the bisphosphonates. Aust Dent J 2009;54 (Suppl 1): S51-61.

[6] American Association of Oral and Maxillo-Facial Surgeons (AAOMS). Position Paper on Bisphosphonate-Related Osteonecrosis of the Jaw-2009 Update. Available online at www.aaoms.org/docs/position_papers/BONJ_update.pdf. Accessed on January 182013.

[7] Marx RE, Sawatari Y, Fortin M, Broumand V. Bisphosphonate induced exposed bone (osteonecrosis/osteopetrosis) of the jaws: risk factors, recognition, prevention, and treatment. J Oral Maxillofac Surg 2005;63:1567-75.

[8] Yarom N, Yahalom R, Shoshani Y, Hamed W, Regev E, Elad S. Osteonecrosis of the jaw induced by orally administered bisphosphonates: incidence, clinical features, predisposing factors and treatment outcome. Osteoporos Int 2007;18: 1363-70.

[9] Marx RE, Cillo JE Jr, Ulloa JJ. Oral bisphosphonate-induced osteonecrosis: risk factors, prediction of risk using serum CTX testing, prevention, and treatment. J Oral Maxillofac Surg 2007;65:2397-410.

[10] Biasotto M, Chiandussi S, Dore F, Rinaldi A, Rizzardi C, Cavalli F, et al. Clinical aspects and management of bisphosphonates-associated osteonecrosis of the jaws. Acta Odontol Scand 2006;64:348-54.

[11] Fedele S, Porter SR, D’Aiuto F, Aljohani S, Vescovi P, Manfredi $M$, et al. Non exposed variant of bisphosphonateassociated osteonecrosis of the jaw: a case series. Am J Med 2010;123:1060-4.

[12] Marx RE. ORN: a new concept of its pathophysiology. J Oral Maxillofac Surg 1983;41:283-8.

[13] Curi MM, Dib LL, Kowalski LP, Landman G, Mangini C. Opportunistic actinomycosis in ORN of the jaws in patients affected by head and neck cancer: incidence and clinical significance. Oral Oncol 2000;36:294-9.

[14] Hansen T, Wagner W, Kirkpatrick CJ, Kunkel M. Infected ORN of the mandible: follow-up study suggests deterioration in outcome for patients with Actinomyces-positive bone biopsies. Int J Oral Maxillofac Surg 2006;35:1001-4.

[15] Støre G, Eribe ER, Olsen I. DNA-DNA hybridization demonstrates multiple bacteria in ORN. Int J Oral Maxillofac Surg 2005;34:193-6.

[16] Donath K, Breuner GA. A method for the study of undecalcified bones and teeth with attached soft tissue. J Oral Pathol 1982;11:318-25.

[17] Favia G, Pilolli GP, Maiorano E. Histologic and histomorphometric features of bisphosphonate-related osteonecrosis of the jaws: an analysis of 31 cases with confocal laser scanning microscopy. Bone 2009;45:406-13.

[18] Pazianas M. Osteonecrosis of the jaw and the role of macrophages. J Natl Cancer Inst 2011;103:232-40.

[19] Hansen T, Kunkel M, Weber A, Kirkpatrick CJ. Osteonecrosis of the jaws in patients treated with bisphosphonates histomorphologic analysis in comparison with infected ORN. J Oral Pathol Med 2006;35:155-60. 
[20] Melo MD, Obeid G. Osteonecrosis of the maxilla in a patient with a history of bisphosphonate therapy. J Can Dent Assoc 2005;71:11-13.

[21] Ruggiero SL, Mehrotra B, Rosenberg T, Enhgroff SL. Osteonecrosis of the jaws associated with the use of bisphosphonates: a review of 63 cases. J Oral Maxillofac Surg 2004;62:527-34.

[22] Lugassy G, Shaham R, Nemets A, Ben-Dor D, Nahlieli O. Severe osteiomyelitis of the jaw in long-term survivors of multiple myeloma: a new clinical entity. Am J Med 2004;117:440-1.

[23] Hansen T, Kunkel M, Springer E, Walter C, Weber A, Siegel E, et al. Actinomycosis of the jaws-histopathological study of 45 patients shows significant involvement in bisphosphonate-associated osteonecrosis and infected ORN. Virchows Arch 2007;451:1009-17.

[24] Bertoldo F, Santini D, Lo Cascio V. Bisphosphonates and osteomyelitis of the jaw: a pathogenic puzzle. Nat Clin Pract Oncol 2007;4:711-21.

[25] Sedghizadeh PP, Kumar SK, Gorur A, Schaudinn C, Shuler CF, Costerton JW. Identification of microbial biofilms in osteonecrosis of the jaws secondary to bisphosphonate therapy. J Oral Maxillofac Surg 2008;66:767-75.

[26] Weinstein RS, Roberson PK, Manolagas SC. Giant osteoclast formation and long-term oral bisphosphonate therapy. N Engl J Med 2009;360:53-62.
[27] Thiel HJ. The ORN: Part I. Etiology, pathogenesis, clinic, and risk factors. Radiobiol Radiother 1989;30:397-413.

[28] Andrews N, Griffiths C. Dental complications of head and neck radiotherapy: Part 1. Aust Dent J 2001;46:88-94.

[29] Reszka AA, Halasy-Nagy J, Rodan GA. Nitrogenbisphosphonates block retinoblastoma phosphorylation and cell growth by inhibiting the cholesterol biosynthesis pathway in a keratinocyte model for esophageal irritation. Mol Pharmacol 2001;59:193-202.

[30] Marx RE. Pamidronate (Aredia) and zoledronate (Zometa) induced avascular necrosis of the jaws: a growing epidemic. J Oral Maxillofac Surg 2003;61:1115-18.

[31] Fournier P, Boissier S, Filleur S, Guglielmi J, Cabon F, Colombel $\mathrm{M}$, et al. Bisphosphonates inhibit angiogenesis in vitro and testosterone-stimulated vascular regrowth in the ventral prostate in castrated rats. Cancer Res 2002;62: 6538-44.

[32] Santini D, Vincenzi B, Avvisati G, Dicuonzo G, Battistoni F, Gavasci M, et al. Pamidronate induces modifications of circulating angiogenic factors in cancer patients. Clin Cancer Res 2002;8:1080-4.

[33] Allen MR, Burr DB. Mandible matrix necrosis in beagle dogs after 3 years of daily oral bisphosphonate treatment. J Oral Maxillofac Surg 2008;66:987-94. 